\title{
Digital participation and collaboration in architectural design.
}

\author{
LAING, R.
}

2018

This is an Accepted Manuscript from an example book chapter published by Routledges in Digital Participation and Collaboration in Architectural Design on 22/8/2019, available online: https://www.routledge.com/9781138062641. 


\section{Chapter 2: Digital technologies in architectural design}

The chapter deals how embedded and emerging digital techniques have been used within architecture, both to help develop ideas in the mind of the 'design team', and to then communicate those ideas to a wider audience. The chapter, though, opens with a wider discussion of information visualisation, in order to make clear the importance of communication, insight and intention within the choice of visualisation method.

This book is concerned with the ways in which digital technologies can be used to both support collaboration between participants in design, and to encourage and support participation among groups who might otherwise not be part of a technical or professional team. Later chapters will discuss and explore why these might be desirable goals, and will refer to a whole range of studies where such issues have been studied. What is important to recognise, though, is that people and engagement are at the heart of this discussion.

A key point when considering any method of visualization, and particularly visualisation of information, is the result of looking at a diagram, picture or other image (Spence 2007). Where that result has been acquired in the mind of the observer, rather than as a result of text or statistical analysis, we can say that 'insight' has been acquired. The examples in section 2 all show how visualisation can be used to illustrate information, and the example of John Snow in particular illustrates how a visually driven approach to understanding a topic led to insights which would have been otherwise unlikely to be derived.

In this sense, we must try to remember that visualisation is essentially a cognitive activity, which is not reliant on computers. Of course, much of this book makes a great deal of the manner in which computers and IT can be used to facilitate the visualisation process. However, we must not confuse this process of visualisation with the aim of the exercise, which is to assist understanding and identify previously hidden facts. Previous research by a range of authors (for example, (Lim et al. 2004, Sener and Wormald 2008)) has successfully studied the relationships between designer, medium used to design, and the resultant visualisations. Such studies have shown that the approach taken to visualisation itself can affect the insights drawn, and that care 
should be taken to match the visualisation method (e.g. sketching, CAD, 3D modelling, physical modelling, painting, etc) to the desired and intended purpose of the exercise.

It is also true that by using visualisation we open up the possibility of juxtaposing information of different types on the same image or model. For example, it should be possible to incorporate details of material and construction design with temporal (i.e. time data) or environmental data (e.g. temperatures, insulation values, and so on). Spence (2007) suggests that we could also categorise such data in terms of whether it is quantitative (or qualitative), ordinal or categorical, and that we can then aim to draw relationships between these.

\section{Key developments in information visualisation}

In recent years, a range of seminal approaches to the visualisation of information have been discussed by researchers within visualisation, information management and $\mathrm{HCl}$ (human computer interaction/ interface design). The rationale for this emphasis can be attributed to the clear, straightforward and illuminating manner in which data was presented, and the extent to which the visualisation process itself then influenced people's understanding of the underlying data. Whilst the examples presented in this section directly influenced a range of discipline areas including history, workforce deployment, health studies, urban planning and transport management, all have resonance in the modern world, and can easily be translated to needs within modern architectural design, urban planning, construction and building maintenance.

\section{The march to Moscow}

Charles Joseph Minard was mapmaker to Napoleon and his illustrative map of Napoleon's 1812 campaign and march on Moscow has become recognised as an important illustration of the manner in which visually straightforward representations of data can be used to convey complex information to the reader.

Minard's map ${ }^{1}$ illustrates a disastrous military campaign, where Napoleon started out with 440,000 soldiers, and returned with only 10,000. The map successfully conveys the size of the army at various points on the route, the temperatures endured, and

\footnotetext{
${ }^{1}$ An open access version is available for viewing via: https://upload.wikimedia.org/wikipedia/commons/2/29/Minard.png
} 
ultimately the extent to which the returning army was a tiny fraction of the original. The reader arguably requires little explanation of the difference between the two colours (journey there and back), and the map in itself serves to entcourage a retention of information about the campaign in a manner which might be more difficult using traditional text and tables. As Spence (2007) wryly notes, though, 'the map might not be entirely suitable as a recruitment poster'. ${ }^{2}$

Some comparison can be easily drawn from how such approaches could be manifest in some typically used approaches to the visual communication of information in construction. For example, critical path theories often used to display and represent information concerning the order, scale and timing of construction tasks take a heavily simplified approach to communication, which although visually distinct from images of 'actual' construction, do well to successfully convey complex relationships between tasks, and resources.

\section{John Snow and the 1854 Cholera epidemic}

A particularly interesting example of information visualisation, from the field of health studies, concerns the well known outbreak of cholera which infected London in 1854. At the time of the outbreak, it was generally thought that cholera was spread through the air, or through person to person contact. Although there was little or no medical evidence to support that theory, the response of authorities at the time had been to encourage those suffering to be kept apart from healthy individuals, although this had little apparent effect on spread of the disease.

Nowadays we are, of course, well aware that cholera is spread through contaminated water supplies, a fact which was made apparent through the visual analytical work of Dr John Snow. Snow mapped the incidence of outbreaks against various physical features within the streets most badly affected, and noted that infections appeared to be concentrated around outside water pumps, used by local residents to supply water for drinking. Snow hypothesised that there was a link between the water supply and the outbreak, and closing off the pumps led to an immediate and very significant drop in the rate of infection. That such a breakthrough was possible in the absence of modern techniques enabling chemical analysis of the supply can be attributed solely

\footnotetext{
${ }^{2}$ Further biographical and other information on Minard can be found via this link: http://www.edwardtufte.com/tufte/minard-obit
} 
to the use of a visualisation technique. Had Snow relied on commonly held medical beliefs of the time, such a breakthrough would almost certainly have gone undiscovered.

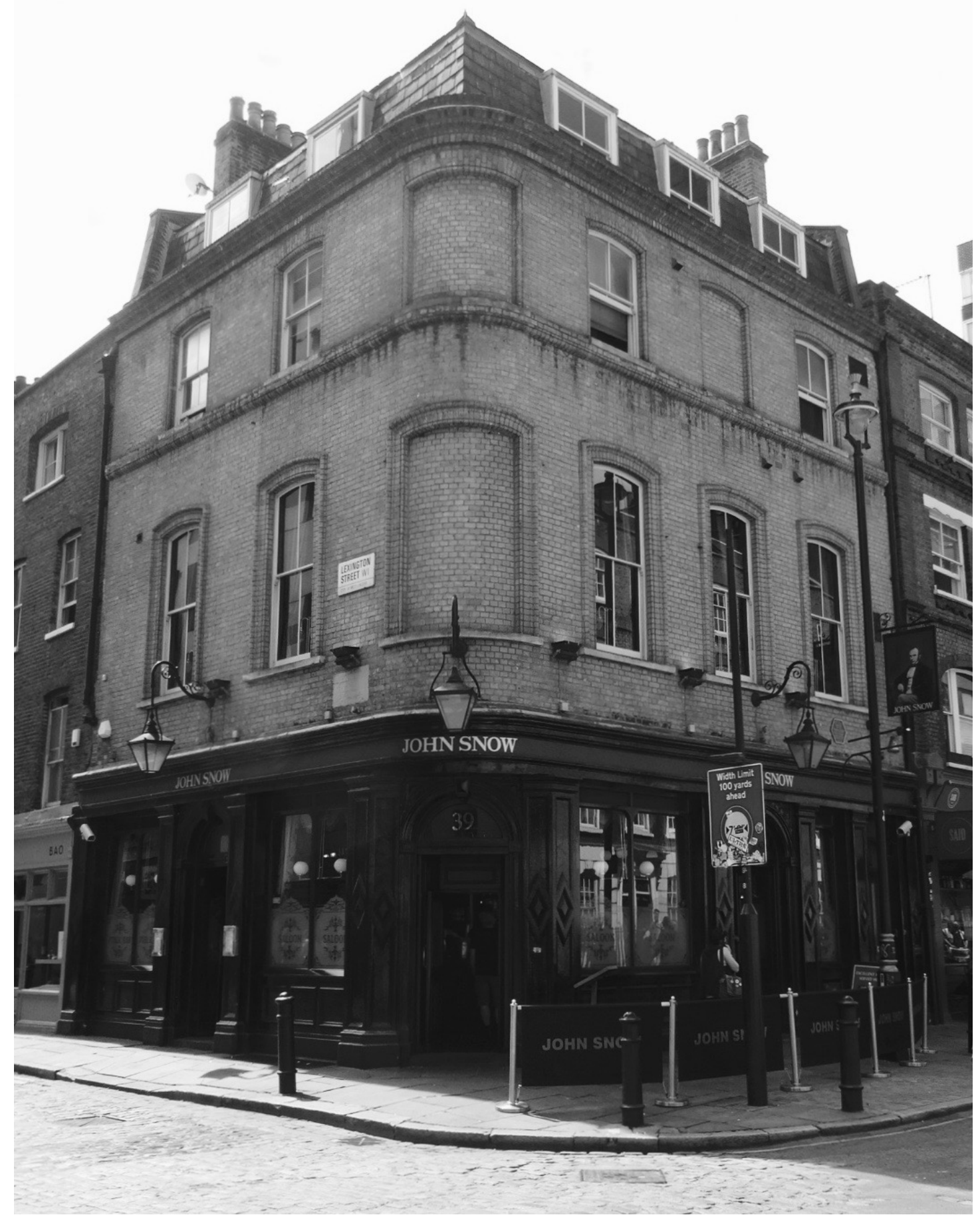

Figure 2.1: John Snow pub, at the site of water pump in Broadwick Street, Soho, London. 
Again, it is possible to draw modern resonances with work in the built environment, possibly most notably in relation to the development of research concerning workflow and ergonomic design of sites (Osman, Georgy and Ibrahim 2003).

\section{Harry Beck}

It is interesting to note that one of the most widely recognised and used examples of design from the $20^{\text {th }}$ century was produced not by a designer, but by the underground electrical engineer Harry Back. Beck worked as an engineer with the London underground system, and recognised that users of the system had no real reason to need to know exactly where the actual routes of each train actually travelled. That is, one can only get on and off trains at stations, so there is no strong transport-related reason to now where the middle of a line might pass under. Early versions of the (pre-Beck) underground map included accurate representations of major streets, and crucially did little to simplify routes for users. Beck's own map made a number of visualisation breakthroughs, including the use of nodes to represent changeover points, and the clear use of colour coded lines to denote each line.

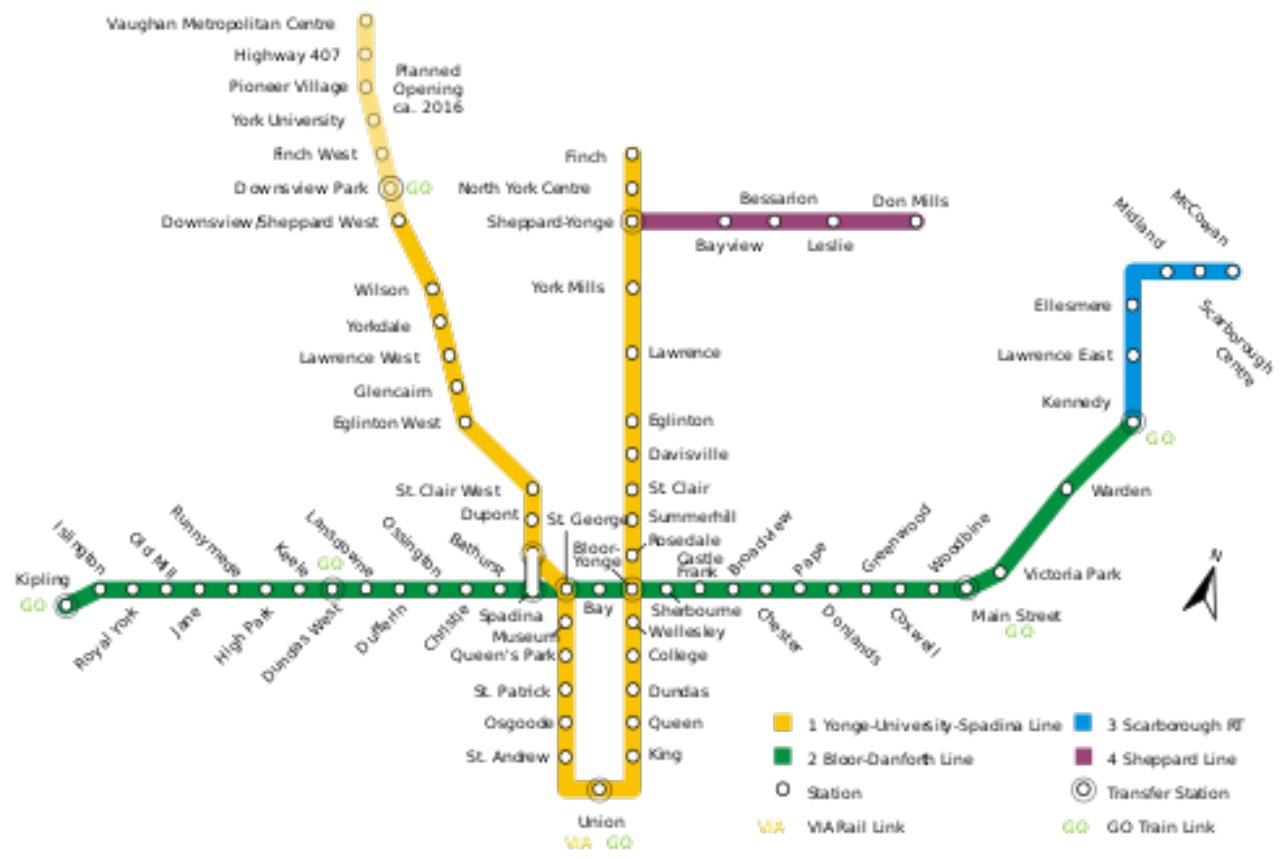

Figure 2.2: Map inspired by Harry Beck's design for London tube map

(https://commons.wikimedia.org/wiki/File:TTCsubwayRTmap-2007.svg) 
Although the river Thames is shown in an abstract form, the map gives little space to real geographical distance, and some stations which appear on the map to be very close, are actually far apart (and vice versa). Beck's map continues to have a huge influence on the way in which most transport systems are represented throughout the world, and its ability to separate issues of actual transport logistics (e.g. time of travel, ticket purchase) has been drawn on by many organisations to suggest anything from a smooth form of managerial operation to subtly suggesting to tourists that countries are smaller than they actually are, thus placing readability and transparency of information over geographical realism.

\section{Visualisation in architectural design}

Recent research has explored the nature of the design team in construction, and methods through which the multi-disciplinary team can work to collaboratively develop ideas and solutions. This has included discussion of variation in terms of working practice between professions, and how this might vary between practitioners and those still in education (Kokotovich and Dorst 2016, Stompff, Smulders and Henze 2016).

One might argue that the field of digitisation including visualisation of designs, data, information and so on, requires technical development to sit at its heart. After all, many of the aspirations of the 1980s to somehow coordinate the information contained in projects has only become possible through the iterative development of computer based systems to allow this to happen. However, one could also contend that this process must be driven by user need, rather than what appears to be technically interesting or challenging. After all, visual methods (through sketches, drawings and so on) have been the main form of communication within the industry for centuries, and moves to incorporate digital tools in the design process cannot and should not ignore that point. Where the two can proceed in parallel, perhaps this represents an ideal scenario, yet requires the design team to be able to convey their own creative and working processes, and for these to be understood and acted upon by software and hardware designers.

Traditionally, architects and urban designers have presented design ideas to clients using static representations such as scale plans and sketches. Architects' plans, although accurate in terms of measurement, rarely give the non-expert viewer a 'feel' 
for what the space will be like when it is finished. It is commonly acknowledged that people often have trouble understanding architects' plans, which can lead to discontentment with the end result (Appleyard 1976). As an alternative or additional method of presentation, sketches are often favoured by architects as a method to present ideas in a more fluid style, and are believed to promote feedback and discussion through their 'unfinished' appearance (Schumann et al. 1996).

While plans and sketches are still the most common means of presentation between architect and client, there is a growth in the area of dynamic 3D modelling. Today's student architects, architectural technologists and designers are taught the use of Computer-Aided Architectural Design (CAAD) as a matter of course, and a plethora of new tools has emerged to make it easier and quicker to both sketch and finalise a design using computers. As a result, dynamic walk- or fly-throughs of 3D models are becoming more common, enabling viewers to visually experience an as-yet-un-built environment. As an extension to this, and perhaps signalling a likely future use of most packages, we normally experience the world via a flow of changing visual images. It can be argued, then, that dynamic presentations of a future environment might be likely to provoke perceptions and reactions closer to those that would be found in the real world, than those that would be provoked by static images.

Through government and industry drives to encourage and support the adoption of Building Information Modelling (BIM), one is often struck by the extent to which an emphasis is placed on people, and how people work and behave. 'BIM' refers to the use and application of collaboration throughout the life cycle of a building, underpinned by shared 3D models, attached to structured data and information ${ }^{3}$.

Thus, discussion about collaboration with BIM should logically lead naturally to much deeper discussions about collaboration in general, and we return again to unresolved debate from the 1990s (Latham 1994, Egan 1998). Similarly, discussion about data sharing deals on the one hand with technical ways to share files and information, yet the emphasis will very often return to tracing behaviour and liability. Nevertheless, such discussion will usually be topped and tailed with arguments which again draw attention to digitisation of processes and ways of working residing at the heart. This is important, and brings us to consider a legacy of digital tools within design, wherein we can

\footnotetext{
${ }^{3} \mathrm{~A}$ wider discussion of what is, and is not, meant by 'BIM' can be found at http://www.bimtaskgroup.org/bim-faqs/
} 
consider the difference between systems which are aimed at supporting design, engagement and participation, as opposed to systems which aim to somehow mimic traditional drawing and representation. Whilst the former can arguably be said to bring new techniques and technologies into play, attempts to replace the pen, paper and suchlike with digital tools requires a different perspective and have followed a different narrative and development process.

Whilst it is certainly useful to remind ourselves of a number of trajectories which have been followed by the industry since the 1990s, we must bear in mind that the paths concerning digitisation, collaboration and participation have not necessarily been followed in parallel, or even with great consideration being given to the connections which might exist between them. Research in the early 1990s (Koutamanis 1993), for example, saw academic discussion moving towards the use of computers within visual representation of architecture, and the potential to significantly extend the ways in which CAD systems could be used to categorise the components and content of digital models, and the computerisation of design information. Within such research, this also encompassed discussion of the digitisation of material compiled and presented in a non-digital format (sketches, drawings, schedules), and the use of CAD systems to automatically recognise key features and components. One might argue that this remains a challenge, albeit one which perhaps affects digital-to-digital recognition (such as in the use of data heavy and highly detailed LIDAR point clouds). We also see discussion (Koutamanis 1993) of the potential for the seamless and planned use of CAD across the design, construction and in-use phases, and again it is useful to reflect on the ways in which this holds resonance in current development and application of BIM. 


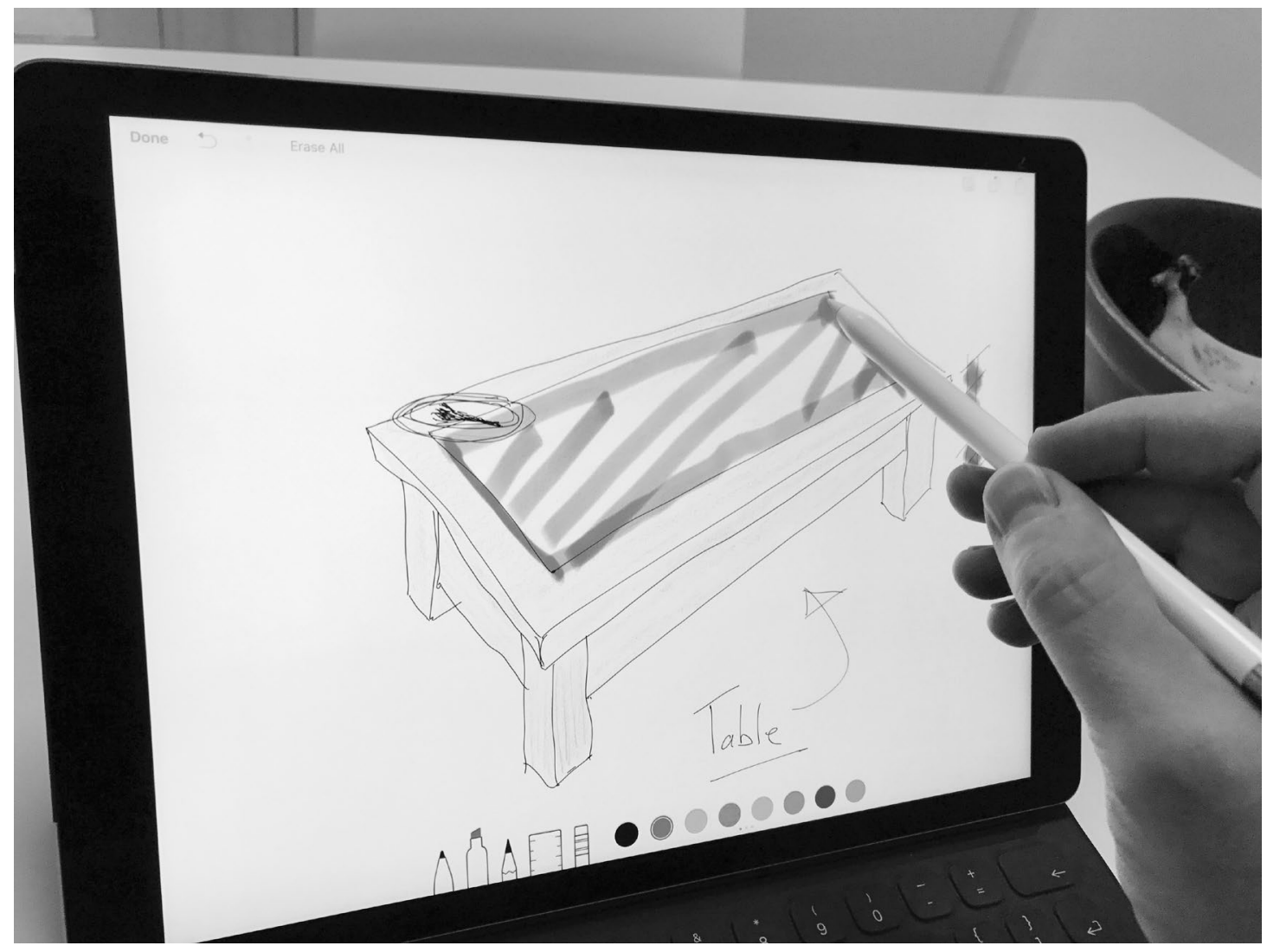

Figure 2.3: Digital sketching

If we refer to many of the discussions regarding collaboration and collaborative practice which were underway during the 1990s (Pahl, Badke-Schaub and Frankenberger 1999), these rarely if ever find a focus within the realm of digitisation, and they very rarely make reference to the use of such technologies as a route to be followed by the industry as a whole. Nevertheless, as technology has evolved and advanced to the point where we can use digitisation to support collaborative practice, it is the aims of the industry which emerged in the 1990s which again return to the fore and become a guiding light for the future development of that technology. Aspirations of the industry, which can certainly be traced back to the 1980s, to ensure that work is coded and presented using hierarchies and taxonomies easily understood across the industry have now started to finally take hold, given that technology has advanced to the point where these processes can be properly supported (with reference again to the drive for Coordinated Project Information ${ }^{4}$ ).

\footnotetext{
${ }^{4}$ http://www.cpic.org.uk
} 
Succar (2009) offered a comprehensive contextual analysis of how both academia and the industry should and could view the development of information modelling in the next few years. Various models have been offered of BIM 'maturity', most of which focus upon the extent to which the digital models are shared (individual, through to federated and centralised models), and the ways in which the data could be applied (e.g. during the life cycle). Succar offered an interesting perspective, though, in that his own framework suggested that maturity could develop thus:

- $\quad$ stage 1 - object based modelling

- stage 2 - model-based collaboration

- stage 3 - network-based integration

In the sense proposed by this framework, collaboration and learning to collaborate is placed at the centre of the path to be followed, and this represents a key hurdle for the industry to cross. After all, the issues of a lack of collaboration and sharing of information and knowledge across the industry are hardly new (Egan 1998), and tools such as those associated with BIM may not, in fact, have been developed to facilitate deep collaboration, even if they are capable of (technically) allowing and (technically) supporting data sharing.

Another slightly different perspective on how building information modelling might be implemented in practice was presented by Jung (Jung and Joo 2011) who concentrated instead on consideration of how digital systems may be implemented across the industry, within individual organisations (for example, constituent members of the design team, the client or the builder). That work also recognised that although industry wide standards may exist, the ways in which these are likely to be implemented and rolled out will also depend on managerial issues and policies. This means, in effect and practice, that although the technical problem of being able to share files between various software packages is probably quite easy to solve, using file types and where possible open source file configurations (Industry Foundation Class, IFC), deeper challenge perhaps exists for all participants in the information modelling process when we start to think about the categorisation of data, such as elements and objects.

Succar (2009) tended towards describing collaboration within building information modelling in terms of knowledge and data sharing. This is a useful lens through which to regard the technical practice of BIM, as it allows the collaborative framework to 
remain focused on the technical capabilities and data sharing characteristics of the three stages (modelling, collaboration and integration), as proposed by the author. 'Knowledge', however, within the context of BIM, tends towards making reference almost exclusively to the information contained within a model, whilst the notion of model-based collaboration is perhaps similarly limited towards the consideration of data interchange between various data file formats. Succar rather successfully set the scene for a particular strand of research exploration, within which we can usefully begin to interrogate some underlying themes. He mentions, for example, the use of visual language within the industry, and a need to explore how this may be developed and tailored to respond to the emerging technical challenges. This is important, and relates to later discussion in this book regarding the use of visual media (e.g. sketching) to develop and communicate ideas.

What I would be inclined to argue at this stage is that much of the digitisation we have seen taking place within the industry over the course of a number of decades has tended to either directly replace existing and established traditional, analogue, tools or sought simply to somehow augment existing practice. What we have arguably seen much less of has been any significant move towards that digitisation representing a form of industrialisation. What sets the object-based and information rich modelling we see within BIM to one side of that digitisation trajectory is that the relationships between aesthetic design and technical aspects of the construction process are far closer than at any stage in the past. That is, conceptual ideas within a BIM environment will be data-rich, and that data can be carried through technical design. However, the nature of many of the software tools commonly used within BIM-enabled design practice means that there is a need to attach technical detail at an early stage, with the implications of those choices evident.

Added to this we have the capacity for a far wider team of participants to contribute to the design and modelling process. One might be so bold as to argue that an industry which has shown itself to be fairly intransigent with regards to collaborative working within the team, but arguably even more so with regards to the active participation of end users, may find it rather difficult to enact significant behavioural change. Therefore, what Succar identifies as a constraint to the meaningful adoption of BIM in practice, namely social phenomena, requires specific attention. In this chapter, we will touch upon some of the digital tools and techniques which have developed over the years, and which may help to ameliorate this process. In later chapters, we will explore practical studies through which it has been possible to study in greater depth the 
impact of digital tools, and the capabilities of those digital tools, when attempting to foster an environment of open collaboration and participation.

The following sections take us through the development of digitisation from the 1970s, with an emphasis placed on technology to be used by the design team, and where an understanding of what is meant by 'the design team' is perhaps narrower than that which would now be accepted and understood by most in the modern construction industry. We will return in later chapters to the notion of collaboration and participation involving a much wider constituency, including participants from outside of the professional industry (including the general public, building users and other stakeholders). For the meantime though we will focus our attention on professions within the industry.

\section{Early uses of digital drawing in architecture}

Although the early development of Computer Aided Architectural Design (CAAD) concentrated on the production stages of projects, the potential for using digital media and $C A A D$ at the early design stages has been recognised for many decades (Eastman 1974, Hyde 1989). 


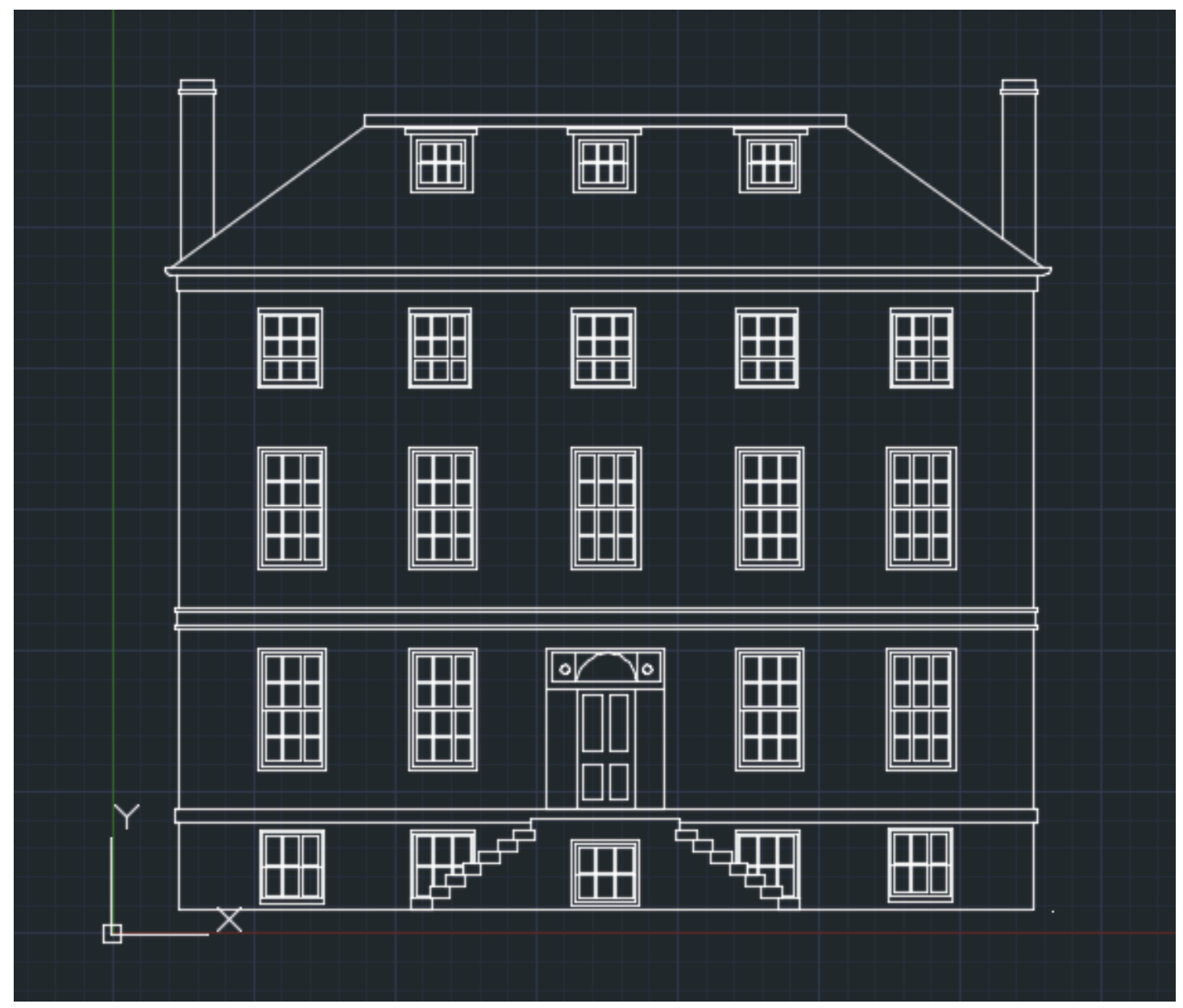

Figure 2.4: Typical CAD output

Eastman, who would later become a highly influential voice in the development and application of BIM in practice, put forward a number of key arguments in 1974, which still hold resonance today. Arguing, at the time, that the USA lagged behind other countries in terms of the application of CAAD, Eastman explicitly associated the use of computer technology in architecture with the theme of industrialisation. He argued that the dominance of small companies in the industry, and a lack of national crises (housing shortage, and suchlike) meant that a widespread demand for revolution of practice had not (as yet) taken place. This was reflected in a relative lack of funding for applied research in the subject area, with the practical application of CAAD difficult due to the large amount of space and investment required to deal with the hardware and data storage requirements. Three areas of research which were being tackled, and which certainly still hold relevance and importance today were:

- the digital representation of space and objects,

- spatial planning (using algorithms, in this early discussion), and, 
- what was described as man/machine communication (typically studied within human computer interaction research, or $\mathrm{HCl}$, in the intervening period).

One could argue that the consideration of how to represent and arrange objects in a digital space has seen the greatest advances, with issues of model viewing, manipulation. Furthermore, issues such as clash detection have taken a central place in the development of architectural software. Where Eastman's early paper still holds most powerful resonance, though, lies in the discussion of $\mathrm{HCl}$ :

- Methods and approaches through which we can find meaningful connections between the digital model and drawings, and,

- Means through which software can make useful interpretations of sketches.

Examples discussed included early digital drawing tools (tablets) and methods through which existing drawn information could be taken into a digital domain (cameras, as opposed to scanners). Whilst this might well have been a basic aspiration in the early development of CAD systems, more recently the move towards models which contain information 'about' objects (beyond their size and appearance) means that there is a need to identify not only basic and 'lower level' information such as vectors and dimensions, but also to be able to recognise (in drawings produced using traditional methods) the higher level components and detail contained within (Lu et al. 2005). This again reminds us of the potential for digital models to be used through the life cycle, but also the fact that digitisation of information will not refer solely to new buildings and new design.

Again, though, Eastman returned to a connection between CAAD, industrialisation and prefabricated building systems as the most obvious route to widespread adoption of such systems, signposting a gap between intellectual demand and technical capability which would not be narrowed until the significant development of CAD/CAM and advanced digital prototyping systems much later.

Complementary research from around the same time (Willey 1976) suggested that there was a need to find connections between traditional approaches to design (such as drawing, physical model making, photo montage, and so on), and that an extension of existing manual techniques (sketching) could drive the development of new digital tools. Willey also described how systems at the time appeared to be geared towards 
either early stages of design, or (and more usually) towards the intensive production of technical design and construction drawings. The centrality of drawing as a key part of the design process, as opposed to simply being a medium to express ideas, was highlighted, as was the importance of an interface which complemented the designers capabilities (rather than driving the process of design per se). The work referred at a conceptual level to the automated generation of sketch ideas, a subject we will return to later when considering the place of simulation within recent CAAD and BIM software.

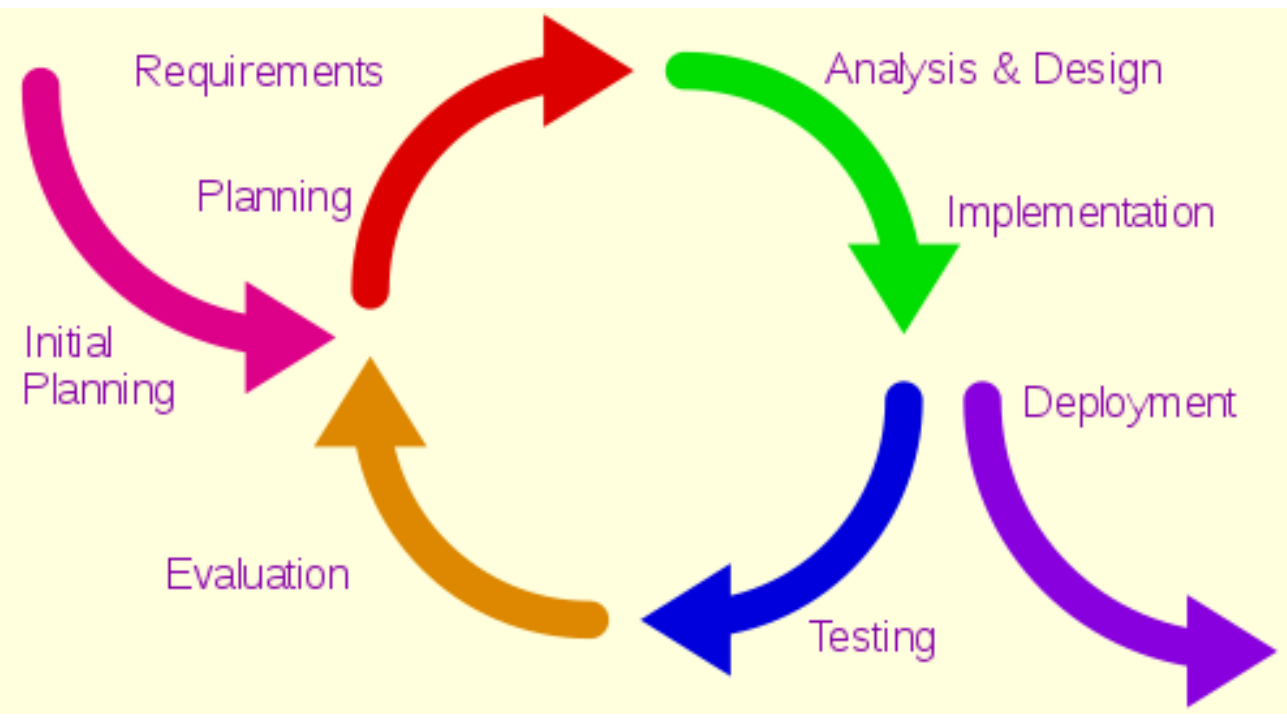

Figure 2.5: Iterative design

(https://commons.wikimedia.org/wiki/File:Iterative development model.svg)

Hyde (Hyde 1989) argued in favour of following a design process whereby ideas are generated through the iterative use of sketching, and where design process was supported by both reason and discovery. Through this, discovery could emerge from the design process itself. In terms of graphical problem solving and visual recording, Hyde argued (through observation of paired experiments) that digital means existing at the time were capable of being used to mimic traditional early stage design processes. The argument was also put forward that CAAD systems offered 'inherent potentials' which were quite distinct to traditional non-digital techniques and tools. However, those potentials would not likely be realised if both the development of the systems themselves and the anticipated design processes did not evolve as a consequence. That is, CAAD would only prove to be truly effective where design processes and workflows utilising CAAD developed and evolved to recognise the inherent qualities of the new tools, systems and skills required. 


\section{Drawing interfaces - drawing, collaboration, communication}

As identified in parts of the preceding discussion, the manner in which a designer and design team are able to interact with a digital system is of vital importance, as the potential to see the system itself leading designs due to a cumbersome or otherwise influential interface is obvious. Research undertaken in the early 2000s (Bilda and Demirkan 2003) identified that some of the benefits of traditional drawing and sketching, including an ability to support perception of 'visual-spatial' features, production of alternative solutions and even understanding of the design problem itself, appeared to dominate when compared with digital modelling tools (that particular study used a CAD interface, with no reported mention of a tablet, or suchlike). We will return to the topic of sketching being useful in design development later, including how this might be effective across groups (van der Lugt 2005, Jonson 2005). Seminal work from the 1990s (Suwa and Tversky 1997) demonstrated the value of sketching within the design process, and how designers are able to benefit from the ways in which sketching can help to 'crystallise' design ideas at the conceptual stages. With regards to the methods through which designers are able to sketch within a digital domain, it is interesting to consider some of the physical interfaces which were developed during the 1970s and 1980s. What is perhaps most obvious with the benefit of hindsight is that many of these interfaces seemed to be directed towards not exactly the replacement of traditional methods of drawing and sketching, but rather to somehow provide a kind of digital surrogate. For example, the development of a early electronic tablets, presumably in an attempt to avoid requiring artists and designers to interface with design software using the keyboard and mouse. In this sense, some of the immediacy of sketching was preserved, albeit in a form which arguably failed to also mirror the benefits of rapid iterative design cycles which are possible when using paper and pens.

Some early studies within the field of design recognised the potential value of using tablets and light pens to mimic the sketchpad, with some discussion of speed and ease of use (Davies Cooper and Cooper 1984), although there were limitations in terms of the ability to find connections between the resultant sketches (useful for presentation) and the main CAD files (Tovey 1989).

What is perhaps more interesting when we consider recent technical developments in terms of the use of digital devices to allow not only two-dimensional sketching, but also 
immersive three-dimensional design work, is the apparent fact that digital tools are now beginning to realise the additional and quite different potential which they offer. We will talk later about the possibility of using digital design methods and their interface with the real physical environment, through the use of CAD/CAM and 3D printing, which brings some kind of logical endpoint to the early conceptual design stages as well as the development of technical design solutions.

One might argue that this does not actually address an important philosophical point, that being the inherent and very important connection between early conceptual design sketches and their continued relevance throughout the technical design stages. Within that particular thread of discussion, we are quite overtly recognising that the medium of design can in fact assist, influence and in some cases even drive the outcomes of the design itself. As we noted earlier, it is also true that visual methods of communication have in fact been the dominant method through which design and technical information has tended to be transferred between members of both the design team and the wider construction and building team. In plain terms, the industry as a whole has always communicated using a visual language, whether through sketching, technical detailing or rendered digital models.

It is interesting to consider how the emergence of immersive digital sketching tools might begin to find a place in the mainstream, but it is also important to consider how the prevalent use of such technology might begin to affect the design process and outcomes. One study (Israel et al. 2009) explored how the inherent differences between 2D and 3D sketching can be understood, arguing that the cognitive difficulties of attempting to represent $3 \mathrm{D}$ environments on a $2 \mathrm{D}$ plane is a challenge to the designer. Of course, one might argue that the education process of most members of the construction design team involves either understanding how a $2 \mathrm{D}$ drawing (conceptual or technical) is representative of a 3D environment or shape, but the third hypothesis of that study - that the sketching process will be influenced by the choice of sketching medium is critical. The results tended to show no benefits of using 3D virtual sketching over 2D sketching/drawing, in terms of creativity and aesthetics. There did appear to be enthusiasm among participants to explore the use of such emerging technology in more depth, though, suggesting perhaps that the approach held potential (at the time, anyway) yet required further development of the interface and tools. Within the context of our discussion, and returning to the theme of collaboration between professionals and with the end users of architecture, we should also consider the ways in which emerging immersive visualisation techniques might to 
be used to support discussion and debate within groups. This strikes one as being important, as the notion of creativity is one of which can and should exist not only within the designer, and the wider design team, but which can extend into a creative application of collaboration and participation in professional practice. Till (2009) refers to the work of Vesely (2004), arguing that 'drawings and other media are seen not as remote abstractions but as the place for the exchange of ideas, information, and inspiration open to all participants in the architectural process'. Till then provides a powerful description of the connections between ideas, narratives and stories, and how these can then translate into or inspire sketches and drawings which will come later in the design process. Elsewhere, Till (2009) draws our attention towards the use of drawing and sketching as a central communication medium, not only within architecture, but extended to the construction industry as a whole. In this, we run the risk of presuming that drawings can at once serve a number of purposes, and equally well in each case. For example, if drawing is effective in terms of representing the aesthetic appearance of the building, could that same drawing or set of drawings be used as part of the production process? Again, we should consider the potential connections which exist between digital design and both CAD/CAM and industrialised production.

Till refers to the apparent ability of drawings to represent architecture in restrained manner, but nevertheless a manner through which the contingencies of architecture are either suppressed or completely absent. For example, Till refers to a freezing of time through the use of various types of image (sketches, drawings and photographs), yet this notion of freezing time is just one example of how emerging digital tools hello the modeller, and to some extent the use of the model, to play with dimensions and characteristics in a way which was not possible, or at least rather difficult, prior to the advent of the digital era.

Elsewhere,(for example, in Ibrahim and Pour Rahimian 2010) one begins to follow an argument where one can see an increasing awareness that tools developed to replicate the visual process of drawing by hand lack both the tangible experience of manual drawing, and can inhibit the design process as a result (by which I would tend to include purely visual 3D digital modelling tools). We begin to see a line of enquiry opening up where the future development of drawings tools and software will be likely to feed off the unique potential of ICT tools: 
- Simulation (including environmental and performance),

- Information modelling and management,

- Surface forming,

- Immersion (VR and AR).

Thus, tools are able to act as a significant extension of the existing suite of methods available to the team. The importance of this when we consider the wider design 'team', potentially including members who approach the creative process in a multitude of ways, is clear.

\section{The design team}

One theme to which we will return in later chapters is that of the 'design team'. The UK CDM regulations ${ }^{5}$ state that a designer is 'an organisation or individual whose business involves preparing or modifying designs for construction projects, or arranging for, or instructing, others to do this. Designs include drawings, design details, specifications, bills of quantity and design calculations'.

Where we now think again about the content, nature and use of BIM in the industry, it becomes clearer that the design team might well be quite wide in terms of membership and scope, but that this breadth of involvement comes with a need to manage design activities. This formalisation of the notion that all participants in a design process be regarded not only as a member of the team, but as design participants in their own right, is of great significance, particularly against the backdrop of an industry wide collaborative working (including the facility to track the nature of that collaboration using digital tools). Earlier work in the 1990s (Whyte 1999) established that the discipline-specific and almost isolated nature of professional groupings and academic training of the disciplines forming constituents of the design team can help to either foster collaborative practice, or could in itself act as an impediment to that collaboration being productive and positive. Whyte also spoke of the need for teams to embrace the group participation method, including an understanding of individual and shared expectations, and the dangers of group dominance by one or more team members. Such an understanding of collaboration and the importance need to embrace the idea

${ }^{5}$ Construction (Design and Management) Regulations 2015, were introduced by the Health and Safety Executive. Also known as the CDM Regulations, they are legally binding, and aim to improve health and safety within the industry. 
as well as the practice of the group participation and collaboration appears to be crucial, yet requires psychological and behavioural commitment on the part of all those involved put simply cannot be satisfied through the use of a kind of digital surrogate. That is, active participation in a group requires a commitment on the part of the individual (or at least on the part of the company employing that individual), and collaboration between design team members requires an associated commitment to making that collaboration work.

This appreciation of design team collaboration must be recognised, at least in the context of current discussion, as representing the central line along which the course of a project can be traced. Of course, the use of shared digital models allows us to find a platform within which we can develop, store and collectively make use of project data. As an example, were I (as the Quantity Surveyor) to alter the specification of an insulation material within a BIM (whether it was a shared or federated model), this could potentially have implications for cost, buildability and even energy performance in the longer term. Of course, the individual made the change could be tracked, and any clashes with other linked models would most likely be identified, but acceptance or non-challenge of the change by others in the team could arguably (and legally) constitute a collaborative and deliberate decision.

Whilst some might regard this to be an alarming situation, might we potentially take a far more positive attitude - that collaboration might become meaningful and desirable where there is an opportunity for the 'team' to contribute in a proactive manner, as opposed to being reactive to the decision and actions of others, after the fact?

\section{Resistance to the use of digital technologies and the importance of drawing}

One objection which has been forcefully presented as evidence against the adoption of digitisation in architecture has been the importance of traditional (both manual and tactile) approaches to the development of designs (although the intrinsic connections between sketching, design and creativity continue to stimulate debate, as in (Bilda, Gero and Purcell 2006b, Scheer 2014, Belardi 2014)). Such methods most obviously include the use of sketching, formal drawing and physical model making, often employed to help the designer formulate new ideas, frequently using an iterative process. One could also argue, of course, that the outputs of such methods are accessible not only to the design team, but also to the lay person, with no requirement 
to have access to professional expertise or to specialised equipment. One can consider in more depth however this can be either facilitated or potentially hindered by the use of digital techniques. The subject of whether, and how, digital methods can be used to assist in the development of design ideas, though, is pressing. As we discussed in the preceding section, many of the early developed methods and techniques using digital drawing appear to be aimed at attempting to somehow replicate or to potentially replace manual methods of representing architecture.

One might argue that digital drafting tools, or even three-dimensional modelling software, tend to produce outputs which have quite a different aesthetic qualities to those other hand drawing, but which actually serve a similar purpose in practical terms. At a very basic procedural level, the results from undertaking a two-dimensional drawing of the building details using a computer are not likely to appear very different to those produced using a traditional drawing board and set square. Likewise, one could argue that outputs from many of the industry-leading three-dimensional modelling software packages produce representations of buildings which are geometrically correct and which to some extent can be modelled to show the likely effects of environmental conditions, such as daylighting and weather, on the appearance of buildings, streets and objects within the design. However, we must also recognise that this shift from manual to digital versions of what is in effect the same process does have an effect on aesthetic quality and likely perception. It might be useful to consider the ways in which the animation industry has been affected by the use of digitisation, in this respect. Although early full length animations (such as those produced by Disney in the 1930s, Lasseter 1987) were produced using entirely hand drawn 'cells', this has given way in time to the use of three-dimensional and almost photorealistic animation becoming prevalent and dominant within the industry (the work of Pixar Studios being an obvious touchpoint). Whilst I would not argue that one approach was superior to the other, surely nobody would argue that the aesthetic qualities of the approaches are identical.

\section{Drawing as a design and communication tool}

When selecting tools for any particular purpose, it is essential that the intended purpose of an activity remains foremost in the selection of methods. As proposed by Lawrence (1993) in the early 1990s, taking the position that sketching, formal technical 
drawing, physical model making and CAD were all available tools, one could posit that design tools (of whatever type) should all satisfy certain principles.

- Account for context specific conditions - location, materials, environmental conditions,

- Reject constraints impinging on the design - planning, regulations, social criterial,

- Encourage dialogue and catalyse discussion of solutions - between designers, and with stakeholders,

- Be able to assist in negotiation between diverse professional, social and aesthetic values and goals - in other words, act as a communication tool,

- Reflect a long-term view, including changing needs of users over time (and presumably environmental concerns).

[Lawrence also noted that tools could be used to critique and understand unintended consequences of design, presumably through simulation].

What is interesting to note, given the time period and development of software and hardware capability in the intervening years, is that some of the concerns raised in the nineties (Lawrence 1993) have been a focus for technical developments, whilst others have become complicated as an unintended result of that process. For example, riding the 'chasm' between viewing models and renders on a screen, and the experience of being in the real world has been the focus of technical development, and the availability and accessibility of virtual and augmented reality systems has improved markedly. However, it is still far from the case that such systems are pervasive across the industry, and Lawrence' wider point about matching the communication method to the intended purpose still holds. 


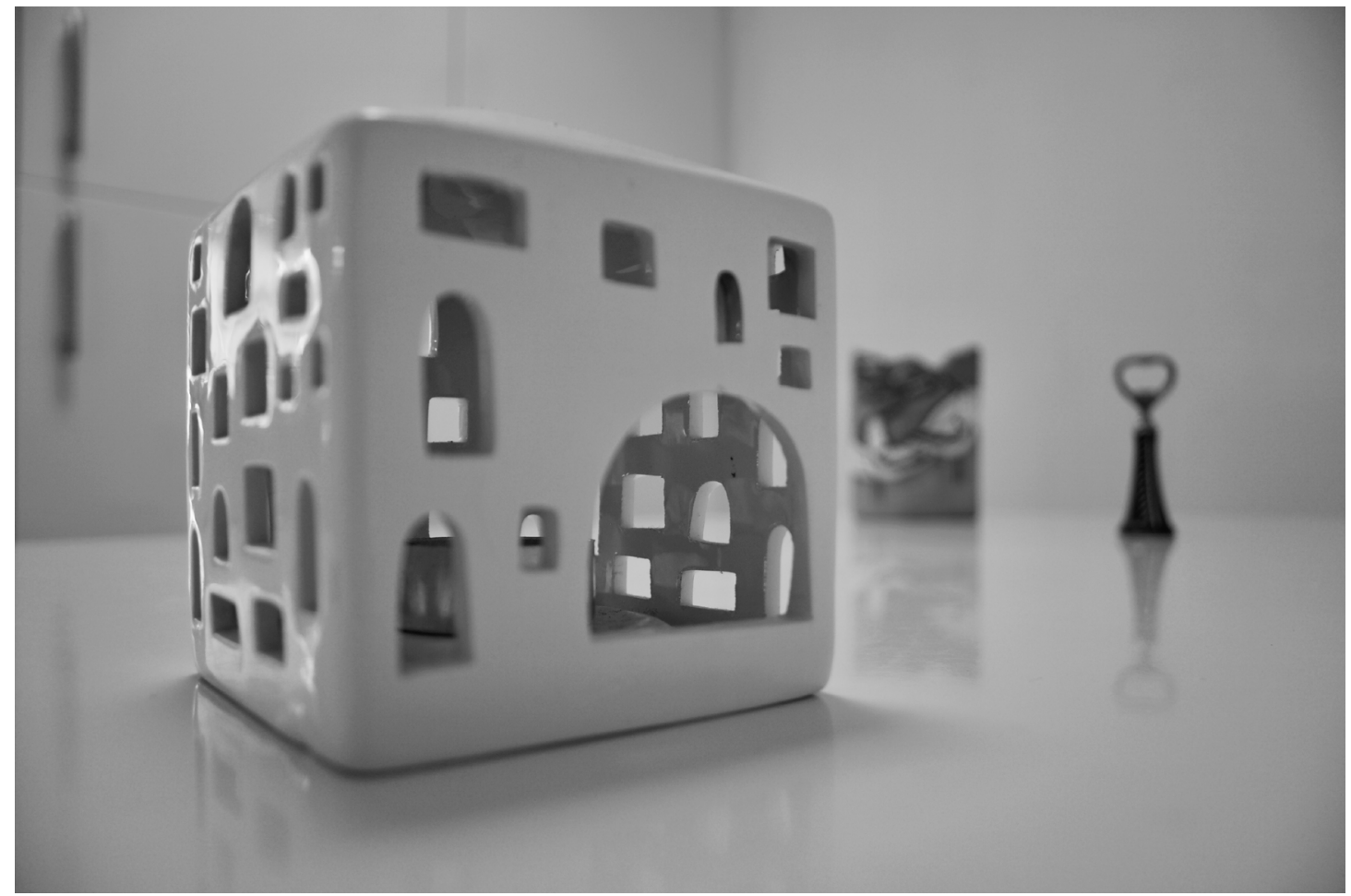

Figure 2.6: Photograph of model with shallow depth of field

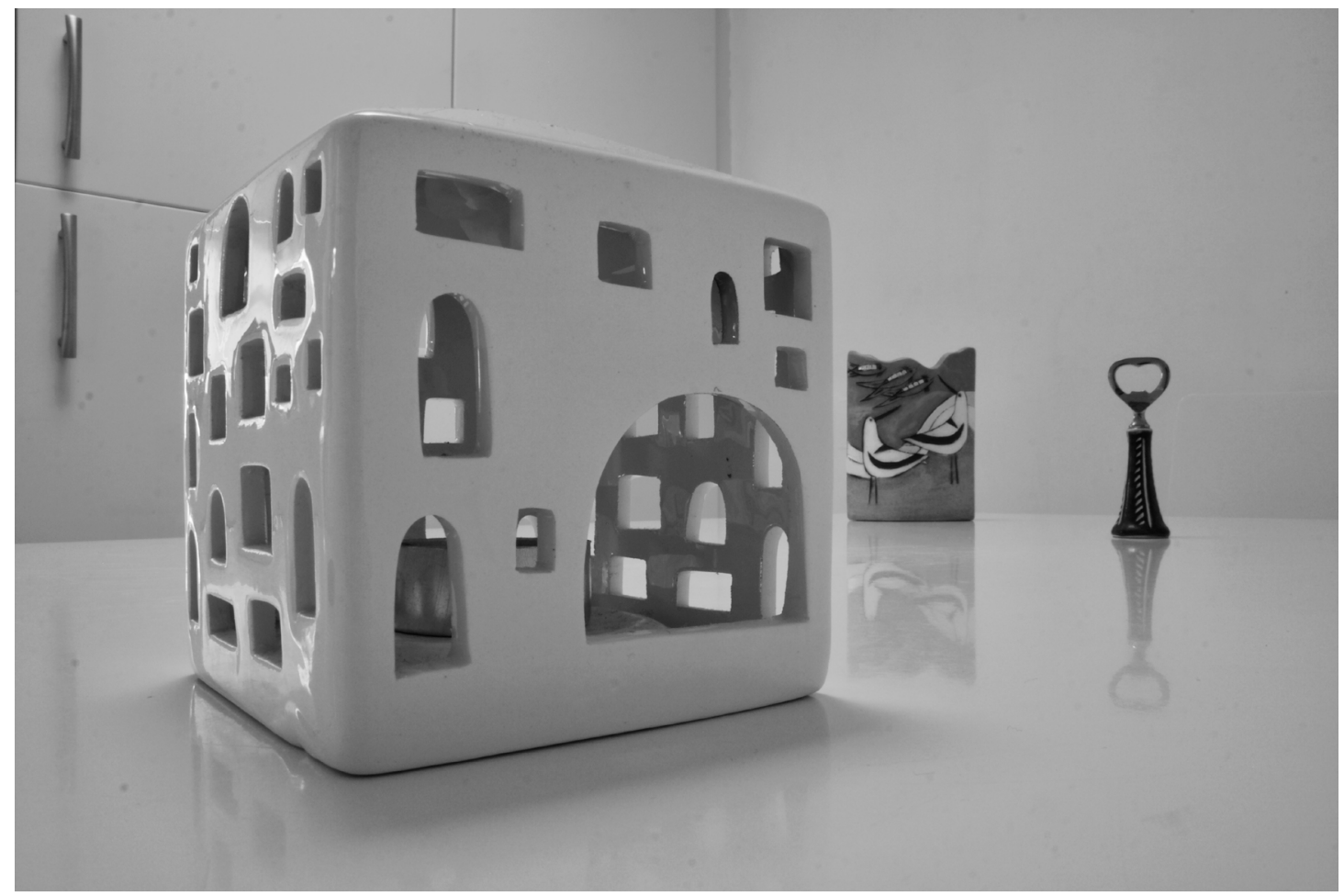

Figure 2.7: Photograph of model with deeper depth of field 
Lawrence also talks about the very activity of physical model making bringing value to the design process, whilst recognising that the intrinsic abstraction of physical models (lacking environmental and human context) means that we must again critically assess their purpose. Certainly, in terms of assisting the designer to develop ideas in an incremental manner, as well as providing a tool for public engagement, physical models remain a powerful communication device. Considering Lawrence's suggestion that the iterative development of a model can help with the clarification and refinement of design ideas, the availability of $3 \mathrm{D}$ printing and rapid prototyping technology (described elsewhere in the book) has had significant effect certainly on the speed with which physical objects can be produced, but also changed the relationship between designer and that object. Material 'printed' to produce the model is intrinsically from the digital domain, and as such may have been developed in a fundamentally different way to models produced using traditional model making techniques. A good example of the importance of physical models in design development is contained in the description of the design process behind Woolf Architects' 'Double House' (Rattray 2003), where great emphasis is placed on returning to early material and model samples.

A well established body of work has been reported in the literature with a specific focus on the importance of sketching within design (van der Lugt 2005). Lugt (2005) noted specifically that sketching carries three main identifiable characteristics and contributions to the design process:

- It supports a cycle of re-interpretation in the individual,

- It supports re-interpretation of the ideas of others, in a group and,

- It allows and enhances access to earlier ideas.

Within other design fields, including graphic design (Schenk 1997), the use of sketching as a core part of the design process has also been recognised as being a skill which designers should value, but is also one which requires a place in the design curriculum. Indeed, the topic of the novice designer, as opposed to the experienced professional, and the ways in which sketching can be used to help the individual explore their own ideas, is well established. Schenk (1997) identified 25 distinct 'types' of drawing within a single study, each with a distinct purpose (e.g. to note information, deal with layout, to express ideas in three dimensions, to demonstrate ideas, and so on), and this nuanced approach to exploring both the production, value and applied purpose of drawings is vital. Indeed, it is likely to hold great value in terms of the future 
development of digital tools if we can understand where traditional tools, approaches and media have existing strength. Rather than attempting to somehow replace traditional analogue methods with digital (for the sake of argument), would it not be preferable to identify where the strengths and potential of emerging digital tools might add something unique, distinctive and useful to the design process? That is, the introduction of digital tools might best be seen as embedding in and being complementary to existing approaches, rather than offering an 'alternative path'.

With regards to accessing earlier design ideas, it is certainly the case that many architectural projects appear to be initially driven by early sketch ideas, and that these ideas remain of critical importance as the technical design develops. Lugt (2005) argues that sketching holds value in itself as a source for idea generation, and offers the possibility of a designer being able to use the sketch medium to think about design ideas as they develop, this being quite distinct to the use of sketching to 'talk' to others and convey design ideas to a wider group. The results from Lugt suggested strong demonstrable connections between sketching and ideas design within the individual designer, but with less strong evidence of sketches providing a strong stimuli for designers influencing each others' ideas through collaboration. This point is interesting, as it perhaps begins to suggest where efforts to study the use and development of tools to connect sketching and the digital domain can be best directed.

Another relatively recent study (Bar-Eli 2013) sheds some light on this phenomenon (the potential to use sketching to share ideas, and its limitations as a collaborative medium) by exploring the characteristics of sketches produced by a range of designers. In particular, that study explored the extent to which this can be useful in itself to inform the design process, and (in the case of that study, which used novice designers) the educational responses which might be enacted. The researchers argued that designers could be profiled as being either learning (recognising the solution generation process as holding education value, perhaps related to the themes of case based reasoning) or designer (with a focus on personal design and design theorisation) oriented, concluding that sketching and sketches can be used in a range of ways to support thinking and communicating and that the ways in which individual designers view and use their own sketches will vary between individuals. The value of using and also understanding sketching and its application by individuals becomes clear, and opens up some questions as to how best to apply this within a collaborative setting. 
Jonson (2005) was interested to explore how the use of digital technology affects the practice of sketching in design (across disciplines including architecture), and found that verbalisation and discussion of ideas appeared to be a greater driver at the very early conceptual stages, and that digital technology appeared to offer considerable potential for enhancing ideation in design (Jonson 2005). In that particular case, a range of techniques were used to capture the design experience (including self reporting, observation and interview), with Jonson identifying that verbalisation of ideas led to more 'Aha!' moments than other design methods and approaches. He noted that all participants regarded the ability to sketch as a skill, and practitioners participating in the study supported the notion that sketching be formally taught within courses.

Elsewhere in the literature (Bilda, Gero and Purcell 2006) we find some evidence to suggest that the use of sketching may not in itself influence the outcome of conceptual design in architecture, although the background of the participants in such studies appears critical. The research was also very clear to stress that there is no suggestion of conceptual designs not existing simply because they are not on paper - far from it, with a recognition that the concepts will exist in the mind of the designer alone (presumably making discussion of those ideas with others difficult, at least in terms of appearance, if not the design intention). Bilda, Gero and Purcell (2006) appeared to show that within a single architect there was very considerable similarity between concept designs produced using a a process of iterative sketching, and designs emerging from think-aloud dialogue, with only the 'final' concept sketched afterwards. The researchers hypothesised that sketching might allow for recording of ideas as they develops and put less 'cognitive load' on the designer. An important point with regards to education was that students learning to design will require sketching not only to help them develop ideas, but to better understand how ideas develop. By comparison, the expert and experienced architects involved in the study appeared able to retain the design process mentally, including associations between elements of the design, and so on. This is in agreement with much earlier work (Suwa and Tversky 1997), which explored how the design cycle, using hand drawn sketching, consisted of a series of iterative cycles, and that the experienced designer was able to deal with longer 'chunks' of design development than the novice. The development of the interface itself also appears to be critical to the success of integrating CAD as part of the conceptual design process (Ibrahim and Pour Rahimian 2010) with evidence that CAD tools designed to support the development of technical drawings can hinder the design process if applied at earlier stages, or by novice designers. 
Following such a discussion, I would tend to argue that the benefits of digitisation in terms of participation and stakeholder engagement are likely to live somewhere other than through the replication of traditional drawing and modelling tools. We could certainly now spent time exploring how architectural drawings may or may not be understandable to the layperson and this is certainly a subject which has been studied to some extent within academia (Lawrence 1983). However, more recent advances in digitisation, including the ways in which the internet can be used to share, develop and debate information of all kinds, is arguably of more interest to the subject matter of this book. It would seem obvious that the ability to develop from and respond to the insertion of data within design models themselves is significantly different to what has come before. It is also arguable that the relatively recent technical ability to share, debate and inform models through the engagement of stakeholders and the layperson represents a significant change in how the industry can operate.

The following sections in this chapter will deal in turn with how models are now moving on from dealing simply with visual representation to becoming models which are dynamic and which can simulate all manner of influences on a design, be these environmental, social or economic. Techniques which can be used to support the communication of such models, both within the design team and to wider audience will then be explored.

\section{Development of ideas through simulation}

In the early 2000s, eCAADe ${ }^{6}$ presented work which explored the key issues facing the future of CAAD within education (Mark, Martens and Oxman 2003). Within that paper, one important question was whether architecture is 'building' or if architecture is regarded as 'design'. This was presented as a pedagogical problem, but can equally be regarded as a positioning question which has become key as the industry has begun a move towards information modelling. It is clearly important and meaningful to think of the design process from a purely philosophical perspective, in that the process itself must engage with important social, aesthetic, environmental and other concerns.

\footnotetext{
${ }^{6}$ eCAADe (Education and research in Computer Aided Architectural Design in Europe) is a non-profit making association of institutions and individuals, founded in 1983 , with a common interest in promoting good practice and sharing information in relation to the use of computers in research and education in architecture and related professions. Further information is available via http://ecaade.org
} 
Indeed, it is many of these issues which underpin and drive the examples we present of practical applications elsewhere in the book. Nevertheless, where digital models are developed in such a way that they contain technical information about components, details, materials specification and the construction process itself, the outcome of work undertaken by the 'design team' is undeniably in the realm of 'building'. There should of course be a seamless connection between the two, but the purpose and application of simulation presents a challenge. What are we intending to simulate, exactly, and how might the outcomes of that simulation be assessed? Can we connect those simulated outcomes back to the aspirations of the designer at the conceptual stage, and begin to explore how the technically simulated outcomes from a technical design connect back to wider social, cultural and aesthetic values?

\section{Information visualisation within education}

One aspect of this discussion which we have not touched upon so far is that of education of the design team. As noted earlier in the work of Eastman (1974) and others, the importance of understanding the implications of CAAD within architectural design and design processes has been recognised since the 1970s, and this has seen some parallel discussion concerning the place of computing within education pertaining to architecture and the built environment (Purcell 1980). Purcell noted that such education would often include both computing hardware and the application of suitable and relevant software, whilst also noting (in 1980) that there was a dominance of teaching relating to computer programming, perhaps with a desire to enable students to engage with simulation of sorts. There was also an interesting distinction made between novice and expert users of CAAD systems, and experts in the development of such systems. That is, a designer wishing to use CAAD tools might have little interest in undertaking programming themselves, but might benefit from collaboration with programmers (with regards to extending software capabilities, or suchlike).

Work undertaken by eCAADe (Mark, Martens and Oxman 2003) in the early 2000s reported that a number of key issues had emerged through both academic research and practice, which would benefit from attention. One aspect of the research at that time dealt with the prior knowledge of students (and presumably staff, across a cohort) with regards to digital skills and knowledge of its capabilities and potential. One might argue (Harrington and O'Connell 2016, Johnson, Gardner and Sweetser 2016) that 
the intervening years have perhaps led to a different situation, where the likelihood is no longer that students will require basic tuition in 3D modelling or even 3D 'design thinking', but that the pervasive use of digital modelling as a recreational pastime will have begun to have an effect on what the student will regard as basic tools of the trade. This perhaps connects with another point raised by Earl et al (2003), in that the practice and protocols with which students are already familiar may require tuition and guidance (although one could also argue that the shift of a professional modeller from visually to information based models is no greater). One could also argue that the digital skills and existing abilities of students entering design education now are likely to outstrip those of existing staff, in some instances.

The connected themes of demonstrating the potential for the use of digital design tools in architecture and the built environment, and the need to understand and recognise how this can present both benefits and difficulties when applied in conjunction with non-digital approaches, is crucial. Within the discussion, Oxman suggested that educators themselves need to understand whether digital architecture is integrated as part of the mainstream, or taught as a separate strand. This connects with points made by others (Jonson 2005, Coyne, Park and Wiszniewski 2002), which suggest that a focus within the development of CAAD software and hardware on production stages of architecture and building constrains the extent to which they have been applied at the conceptual and ideation stages, and the extent to which we can benefit from the intrinsic ability of new devices and approaches to catalyse new practice and theory. Likewise, design strategy should explicitly consider whether design processes are confined to traditional approaches, but in any case should explore and understand these as a core part of the educational design process.

Within the education of architecture and architectural design, teaching has traditionally taken place within a studio environment. Also thinking from the point of view of the history of how studio teaching has taken place, this has tended to mean tuition happening in a physical studio environment, although the past decade or so has seen such a rapid evolution and change with regards to multimedia and the possibilities of virtual collaboration of design teams that there now exists the opportunity to integrate such technology within the studio environment. (Pektaş 2015) noted that terminology concerning the use of a virtual design studio emerged in the 1990s (Wojtowicz 1995), and became the focus for a study, with research concerning the use of the internet, massive multi-user online platforms and the increasing use of virtual and augmented 
reality to convey architectural design. Pektaş (2015) posed a series of important research questions, against three key themes:

- The sociocultural context of design education - the need for designers to appreciate the connected importance of technical, social, economic and other factors, and learn to systhesise these within design.

- The need for a theoretical framework, with regards to virtual design studio that is, to have a framework for teaching not only the technical approaches to virtual design, but to foster and promote a deep understanding of how such working practice might rely on social and interpersonal skills, perhaps complemented by but certainly integrated with technical solutions.

- Student opinion and reaction to changes in studio environment.

Referring to the seminal writings of (Schön 1983), Pektaş reflected on the importance of face-to-face social interaction within the studio environment, but with an informed understanding of how this might bring value to the design process. That is, where the virtual studio itself is designed in such a way that it can support interaction between designers, and support rather than stifle intuitive design and creativity, this may in fact open opportunities which have not been available previously. It is also interesting to read and reflect on the idea that the use of a design studio in education has a basis in both the need to develop skills within students and also a need to simulate work in practice.

Pektas (2015) then continues to suggest that a virtual design studio should attempt to both blend traditional approaches to design with new technology, whilst also benefiting from the apparent affordances of those technologies. Within such a framework, there would appear to be opportunities to augment the intelligence of the design team through:

- Blending of traditional and online techniques (allowing for teams which can be partially co-located, but this not being a constraint to membership)

- Combining numerous tools, making the importance of sharing data, ideas and outputs between tools important

- Supporting the use of cloud computing, including provision of access to external information

- Using online social media to facilitate discussion 


\section{- Guiding participation}

Both the use of cloud computing, and the use of guided participation and collaboration (perhaps using design protocols) has been studied elsewhere (Leon et al. 2014), and tended towards demonstrating that a process (protocol to be followed or mediated) is required to closely consider the use of technology, to ensure that it is in fact helpful to support the progression of design, and does not suppress the potential benefits of using technology in first place.

Previous work (Rohrmann, Bishop 2002) has demonstrated that while computer simulations can obviously only approximate reality, they are acceptable to most people as valid representations of the real world. Other research (Heft and Nasar 2000, for example) found that although studies of environmental perception and aesthetics has traditionally been conducted using photographs or slides, reactions to static displays do not parallel those of dynamic displays. Interestingly, they found that preferences were significantly lower for dynamic compared to static conditions. This finding has implications for the way in which architectural and urban designs are presented to people - a building may look appealing in sketch or CAAD model, but 'using' a building means that we do not experience it in static form.

Bearing this in mind, it is useful to reflect on the definition provided by (Chen 2005) for the subject of information visualisation:

'as visual representations of the semantics, or meaning, of information. In contrast to scientific visualisation, information visualisation typically deals with non- numeric, nonspatial, and high-dimensional data.'

This definition is useful in that it makes very clear the implication that information visualisation is different in intention and methods from approaches typically used to represent 'scientific' data. For example, data concerning the bearing capacity of a particular material will typically be numerical in nature, and might be easily represented using fairly mundane and very familiar visualisation techniques such as histograms. Whilst one might hesitate when considering the visualisation of all data concerning the built environment to remove the possible inclusion of such data (or data concerning cost, for example) from our discussions, we should also try to consider how nonnumeric, spatial and dimensioned data, such as design concept ideas, or social impact data, might be represented in a manner which will be understood by those who need 
to participate in the planning, design, construction, maintenance or other processes affecting a building or area.

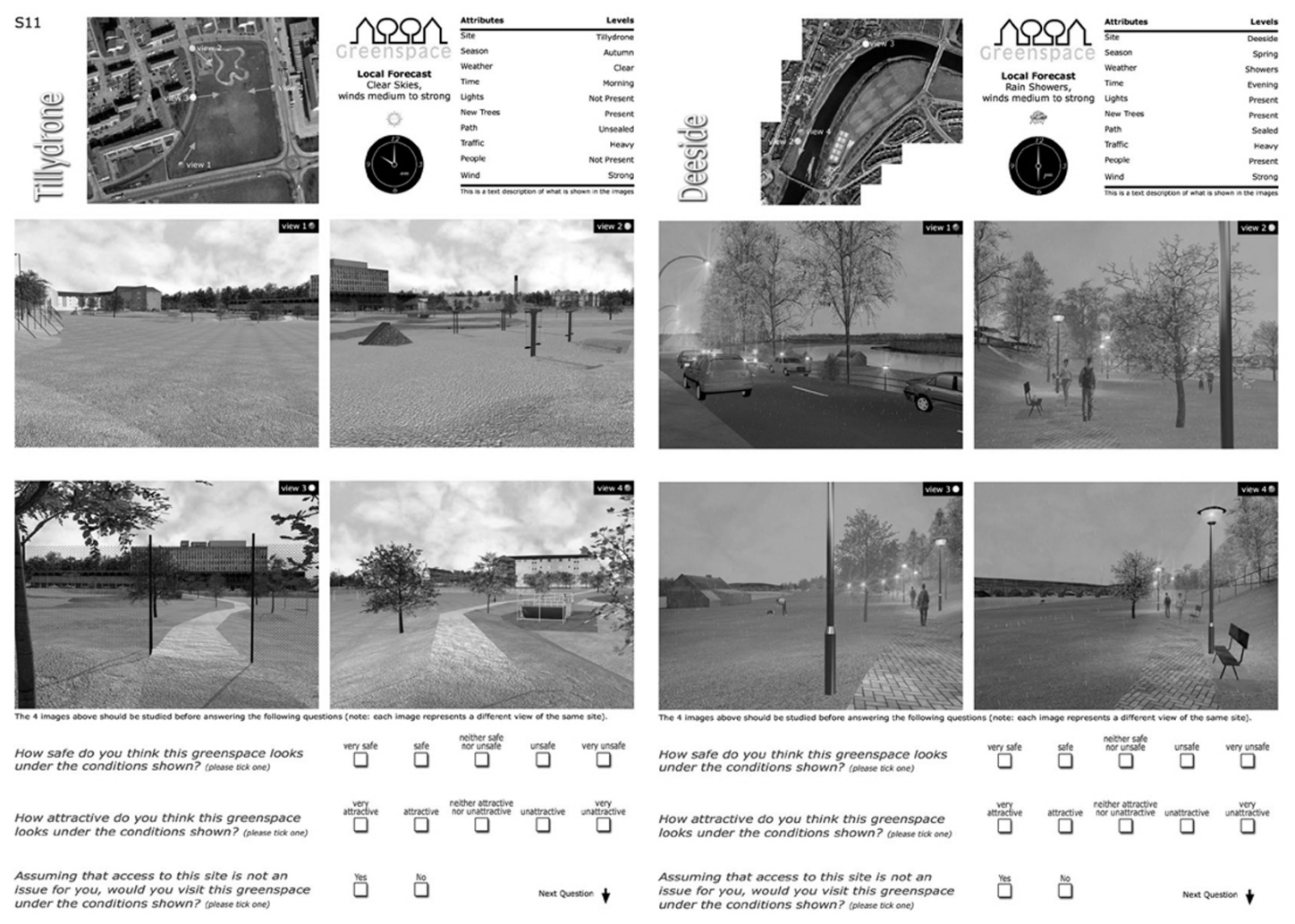

Figure 2.9: Visualisation of Greenspace using combined image and text (Image produced by Stephen Scott.)

Aspects of the foregoing discussion tended to emphasise the potential for the use of digital technology as a part of design, and to provide an effective and useful part of the design outcome. Other studies have also identified the potential for using 3D digital models to convey ideas and concepts, engage students and lead to deeper understanding. Examples certainly abound within heritage studies (Bustillo et al. 2015), taking us back to a consideration of digital visualisation as a communication tool. One could argue that the combined use of photogrammetry ${ }^{7}$ and scanning most certainly holds value and potential within teaching, even when dealing with derelict or debilitated structures, and that this is likely to gain greater value still when combined

\footnotetext{
${ }^{7}$ Where photogrammetry provides the means the create 3D surface models and extract relative proportions from photographs of an object, as opposed to on-site measurement, scanning or the like.
} 
with social and cultural historical study. The study presented by Bustillo (2015) applied methods of digital virtual reconstruction, but in combination with study of history and art with the goal to stimulate interest in both the core subject matter and the technical method being applied.

Chen's (2005) suggested challenges facing the visualisation process, nevertheless, have a broader use, and serve to focus some consideration of the subject matter.

- Usability

- Understanding elementary perceptual-cognitive tasks

- Prior knowledge

- Education and training

- Intrinsic quality measures

- Scalability

- Aeasthetics

- Paradigm shifts towards dynamic interpretation of data

- Causality, visual inference and prediction

- Knowledge domain visualisation

Whilst not all of Chen's key issues appear to have an immediate impact on the design and use of visualisation packages in the built environment, all do in fact have implications for the manner in which we might use such packages in the future.

\section{Usability}

A commonly discussed thread of research, and the first of Chen's challenges, concerns that of usability. This is related to interface design, but is also deeply connected with concerns over the purpose of a software package, and the likely user of the software. Laing et al. (2007) explored many such issues, with particular reference to the use of an interactive model of a historic built environment. That study confirmed that the user's prior knowledge and expectations were vital components of how a system would be used. There were also important human-computer interface $(\mathrm{HCl})$ questions, which require consideration when dealing with moving images, including those of perception of scale and user comfort. 
A common issue affecting many early examples of CAAD packages was that the packages themselves had an over-reliance on geometrical accuracy, which did little to foster their use at the earlier stages of the design process. Similarly, methods commonly used to present planning solutions to members of the lay-public include topdown plan views and construction details - methods of communication not typically otherwise found outside of technical circles.

Therefore, we should consider how the extent to which a package is 'usable' will vary not only between packages, but also between users and between intended tasks. Any particular CAAD system might be perfect for one task, yet entirely unsuitable for another. For example, within my own research we made extensive use of 3D Studio to produce visual representations of street and landscape environments, allowing us to produce rendered outputs. However, we would not have been able to output technical detail drawings directly from the models, or embed elemental data. The implications of this become important as we now move towards an industry where there is potential for domination of 'simulation software' and BIM, where multiple disciplines and individuals will be required to interact with virtual models, and with each other using IT-supported means.

\section{Understanding elementary perception-cognition tasks}

Until very recently, it has been usual for software packages used within the architecture and engineering industries to be aimed at and used by single discipline groups. Although participants will usually constitute part of a larger group, or design team, problems of interoperability between users have abounded for many years.

As multi-user object oriented packages become more popular, it is likely that numerous members of a multidisciplinary design team will find themselves sharing and working together on the same virtual model. Although such a situation has been common within engineering and oil and gas sectors for some time, this is not the case within smaller scale built environment projects. Consideration needs to be given to the manner in which such a potentially disparate group of skilled team members can be enabled to understand the visualised data, which might include information pertaining to materials, cost, supply chain, structure, planning and other issues. The ways in which team members interact with a model will be informed by their familiarity with software, as well as their own discipline and pragmatic requirements. Of course, there is a further 
expectation that longer-term use should be made of such models, requiring that participants not involved in the original study be required to use and manipulate a model to support building maintenance.

The manner and form in which data is presented must be properly considered at the software design stage, to ensure that all members of a team 'read' the same meaning into data presented to them. Chen's (2005) theoretical example showing a 3D dataset could easily be used to illustrate data concerning critical path analysis, or a supply chain. It is vital in such cases that capabilities of software are used to increase clarity and to ensure that all users are clear as to the intended meaning.

\section{Prior knowledge}

In relation to visualisation in the built environment, the issue of prior knowledge takes different forms and has different implications depending on the situation or the intention of the project. To focus initially on prior knowledge of a software package, familiarity with the interface and meaning of the output is clearly essential for all core users. That is, those members of a design team who may be required to interact with software will be required to understand the software's operation, and also the manner in which information can be retrieved, displayed, printed and otherwise exported (to other packages, for example).

This is a subtly different situation to that facing a design team exploring design issues with a client, or with affected members of the public. Issues of site topography, materials, texture, spatial layout and the experience of visiting a real place are arguably almost impossible to convey effectively to the layperson using traditional 2D plans and elevations. In such a situation, prior knowledge of a site on the part of the client would enhance the communication process, if only to provide a context in terms of the existing site and surroundings. In such cases, it may well be the case that detail showing the position and relative size of existing landmarks (e.g. prominent nearby buildings, major roads, open spaces, etc) may in fact be as important as providing detailed information on a new development. That is, prior knowledge in such cases may demand that the impact of a design on a known environment will be vital.

\section{Aesthetics}


With many built environment projects, it is very much the case that the site and project will be difficult (aesthetically) to represent in total photo realism, due to the complex nature of built and constructed spaces. Quite apart from the dynamic nature of built spaces (reference to Chen's 2005 plea for 'dynamic representation' could be useful), where movement of participants and traffic is central to the experience of a place, it is also true that aesthetic preferences are driven as much by constituent parts of a scene (Laing et al. 2006), as they may be by an overall impression of 'beauty'.

These issues may prove useful when considering the value and applicability of various techniques, methods and theories within your own work. However, you should also remember that Chen himself suggests that the list is certainly not comprehensive, and that other issues and fields might be or become equally important in the future.

The purpose of any form of visualisation or modelling will often be to elucidate information or a problem. Particularly within architecture and other built environment disciplines, models will be used for a range of tasks from the design stage through to life cycle management. These stages may be related in terms of the facility or building, but might require quite different approaches to modelling and to data storage. Rather than concentrate immediately on each stage of the 'process', it might be more useful to instead consider the range of purposes to which models may be put.

Firstly, and most obviously, models of buildings can be used to represent the likely final appearance of that building. Abstract models lacking 'detail' of the final materials tend to be commonly used at the conceptual stages of the design process. Such models are useful as they provide the design team with a common object through which ideas can be discussed, developed and debated. Although some studies have tended to show that $2 \mathrm{D}, 3 \mathrm{D}$ and moving representations tend to be perceived by experts and non-experts differently, there is also a strong argument that such 'abstract' models can be used to discuss design solutions with a client at the outset, thus framing what may be the large issues (e.g. spatial layout, overall structure and form) without becoming distracted by details of where each part of the budget may reside. One could also argue that the financial impact of a design may in fact be deeply wedded to the structure and overall form of a building design, and that even abstract models can be utilised to illustrate where aspects (such as service location or room layout) could be altered to drive the design towards a more efficient solution. 


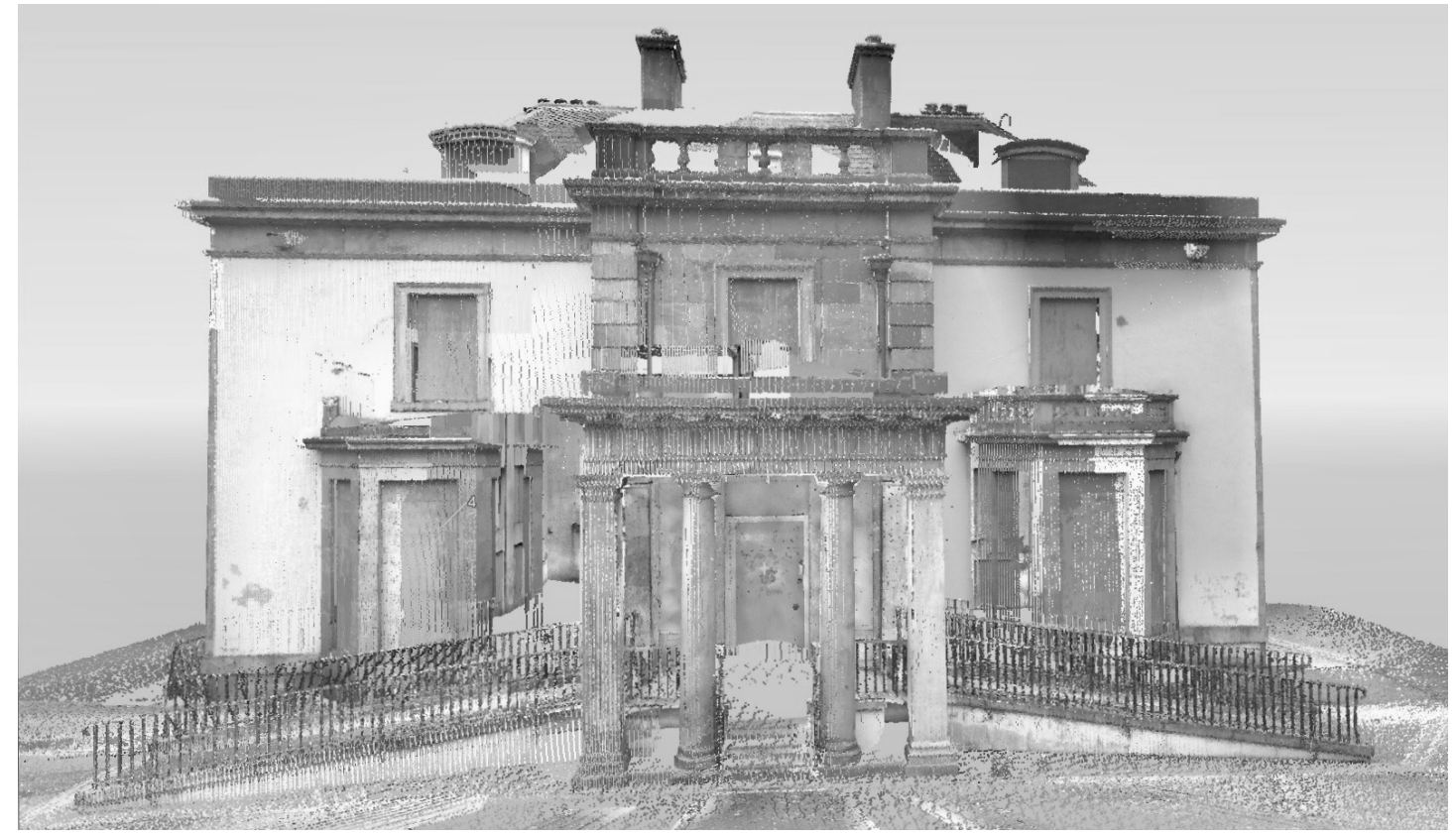

Figure 2.10: Elevation views from laser scan data (Image produced by Dr Marianthi Leon.)

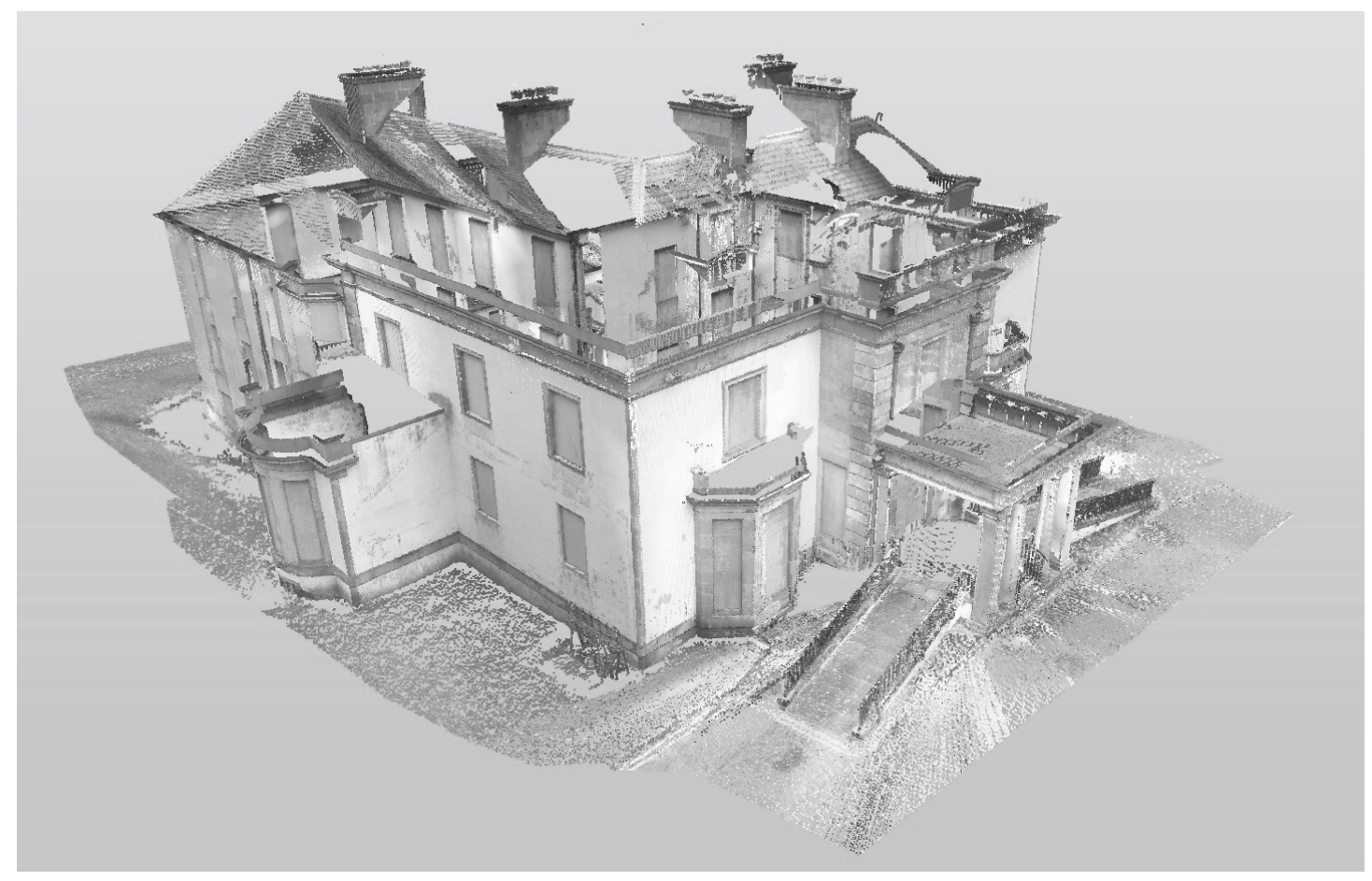

Figure 2.11: Perspective view from laser scan data

(Image produced by Dr Marianthi Leon.) 
This in some ways brings us back to consider the subject of $2 D$ as opposed to $3 D$ representations of space. Although much of the emphasis now placed on the description of development of new computer modelling systems is focussed on the use of $3 \mathrm{D}$ methods, it is certainly true that many examples of historical cutting edge information visualisation involved 2-dimensional representations of space or concepts.

Where modelling has more recently been undertaken in 3-dimensions, using IT based systems, the practical benefits beyond those associated with pure aesthetic consideration of a space have become apparent. Although this can in some ways relate to the topic of prototyping and virtual testing, the increasing availability of systems which are based around object-oriented modelling have allowed designers to produce models which capture not only the appearance but also the physical characteristics of a structure.

A further development in recent years, which certainly relates also to the growth of GIS (geographical information system) within planning and design, is that of $\mathrm{nD}$ modelling. By this, we refer to models where the data contained within extends beyond geometrical data, to include for example scheduling, costing, accessibility, crime, sustainability, maintainability, acoustics and energy simulation (Aouad, Lee and Wu 2005).

Where the model produced to assist with the design and construction of a building is sufficiently detailed to capture all aspects of the building, there is a strong argument that the model should be regarded as a valuable asset to be drawn on during the life cycle. This is particularly true of any model which purports to contain information regarding materials and their performance. Such data is clearly useful at the design stage to support analysis of initial construction cost, but could equally be used to drive an analysis of life cycle concerns include maintenance, replacement and energy costs.

Theories of information visualisation have developed over the past 20 years, and that development has taken place at both the theoretical cross-discipline level, and also within disciplines at the level of application and understanding. Before we become overly concerned with the extent to which computers and IT can influence the way in which we develop ideas of visualisation within current built environment projects, it is worthwhile to take a step back and consider how 'visualisation' can and has been used to successfully present information and data in a manner which is illuminating and 
immediate to the user. An appreciation of such approaches then allows us to appreciate how successful design of the often IT dependant approaches taken nowadays in the built environment relies on a more basic understanding of visual communication to ensure success.

\section{Summary}

In this chapter we began to draw together a number of different strands of discussion. Importantly within these were themes central to information visualisation, where are we could trace important and seminal examples of the use of visualisation and visual communication to convey complex ideas and situations in a manner which was widely accessible. These themes and different practices are important, as they serve to show us how the choice of communication method can have a significant effect on the insights which can be drawn from the underlying information. Thinking for a moment about our earlier discussions of drawing and sketching, we are again reminded of studies which have demonstrated important truths about the use of visual communication to help both individuals and teams to develop their ideas, and to do so in a collaborative manner.

We also began to think in more depth about the notions of collaboration, and how these might be supported by or even stimulated by the use of digital technology. Within the consideration of BIM, it was interesting to note how some models of the maturity of use were almost defined by the extent to which participants in the design process were engaged and taking part in active collaboration.

Later chapters deal with various examples of emerging technology, and consider how these might be brought to bear to help support collaboration within teams, as well as supporting enhanced levels of user participation in design. It is important that we bear in mind these principles of communication and collaboration as we now move on to consider how they may be applied within practice. 